\title{
High glucose promotes tumor cell proliferation and migration in lung adenocarcinoma via the RAGE-NOXs pathway
}

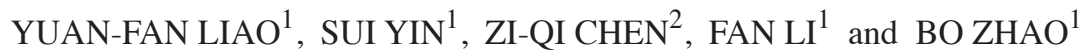 \\ ${ }^{1}$ Department of Thoracic Surgery, Tongji Hospital of Tongji Medical College, \\ Huazhong University of Science and Technology, Wuhan, Hubei 430030; ${ }^{2}$ Department of Clinical Medicine, \\ School of Basic Medicine, Zhengzhou University, Zhengzhou, Henan 450000, P.R. China
}

Received November 1, 2017; Accepted March 19, 2018

DOI: $10.3892 / \mathrm{mmr} .2018 .8914$

\begin{abstract}
Over the past few decades, it has been demonstrated that hyperglycemia can promote lung carcinoma growth, potentially through significantly increased glucose metabolism; however, the underlying mechanism remains to be fully elucidated. In the present study, treatment with a high concentration of glucose (HG) significantly promoted the proliferation and migration of A549 cells. Receptor for advanced glycation end-products (RAGE) has previously been demonstrated to be associated with diabetes mellitus and oxidative stress, and nicotinamide adenine dinucleotide phosphate oxidases (NOXs) are considered to be initiating factors of oxidative stress. Therefore, an MTT assay, wound-healing assay, quantitative polymerase chain reaction and western blotting assays were used to analyze the RAGE-NOX-4 pathway and to determine its potential involvement in glycometabolism-associated tumorigenesis. The present study demonstrated that HG could increase the protein expression of RAGE and NOX-4, whereas the inhibitor of RAGE (anti-RAGE antibody) could suppress this effect. Futhermore, the inhibitor of NOX [diphenyl iodonium chloride (DPI)] could reduce the protein expression of RAGE and NOX-4. Furthermore, inhibition of RAGE led to the downregulation of vascular endothelial growth factor (VEGF) and hypoxia-inducible factor-1 $\alpha$ (HIF-1 $\alpha$ ), thus suggesting that HG may influence angiogenesis and tumor metabolism via the RAGE-NOXs pathway. The present study also demonstrated that the RAGE-blocking antibody downregulated NOX-4 and subsequently reduced the production of downstream
\end{abstract}

Correspondence to: Dr Fan Li, Department of Thoracic Surgery, Tongji Hospital of Tongji Medical College, Huazhong University of Science and Technology, 1095 Jiefang Avenue, Wuhan, Hubei 430030, P.R. China

E-mail: tjhtsdrli@163.com

Key words: high glucose, diabetes, lung carcinoma, proliferation, migration, receptor for advanced glycation end-products, nicotinamide adenine dinucleotide phosphate oxidase-4, vascular endothelial growth factor, hypoxia-inducible factor- $1 \alpha$ inflammatory factors, whereas DPI did not affect the mRNA expression of RAGE but it did reduce the protein level of RAGE and then attenuate the inflammatory response. These results indicated that inhibition of RAGE or NOXs may promote the reduced expression of VEGF and HIF-1 $\alpha$, and NOXs may be downstream targets of RAGE, thus indicating a HG-RAGE-NOXs-VEGF/HIF-1 $\alpha$ association. Furthermore, the results indicated that HG may serve a role in the development of lung adenocarcinoma, mediated by the RAGE-oxidative stress pathway; therefore, the regulation of this glucose-associated pathway may be a promising novel direction for oncotherapy. However, while certain antidiabetic agents have been verified to exert inhibitory effects on tumor growth, they can also have long-term adverse effects on the body, which may limit the value of these drugs as anticancer treatments. In conclusion, the present study suggested a novel attempt to suppress glucose-induced tumor growth using a RAGE inhibitor such as soluble RAGE while avoiding the risk of glucose fluctuation.

\section{Introduction}

Lung carcinoma is a prevalent disease, which is associated with a marked impact on global health and an increasing rate of incidence (1). Tobacco smoking and environmental tobacco smoke, genetic factors, air pollution, and certain occupational exposures, including to asbestos and radon, are considered high risk factors for lung cancer (2-4). Accumulating evidence has suggested that there is an association between diabetes and lung cancer (5-7). In certain clinical trials, the combination of diabetes and lung cancer has been associated with an elevated mortality rate (8), whereas antidiabetic medication can improve the survival rate among such patients (9). In vitro, a high concentration of glucose (HG) has been demonstrated to promote the invasion and metastatic potential of A549 cells (10). These data indicate the potential association between diabetes and lung cancer.

Receptor for advanced glycation end-products (RAGE, also known as AGER), the ligands of which were initially identified as advanced glycation end products, serves a role in diabetes mellitus. RAGE has been reported to be downregulated in non-small cell lung cancer (NSCLC) (11-13), which differs from other solid tumors, including liver cancer, which exhibit high levels of RAGE (14). In the present study, the 
potential role of RAGE in the effects of HG on A549 cells was investigated.

Nicotinamide adenine dinucleotide phosphate oxidase (NOX)-4 is a member of the NOX family, which is associated with generation of endogenous reactive oxygen species (ROS) and mediation of inflammatory responses (15). The NOX-4 protein may also be an intermediary agent in the association between RAGE and inflammation, which may influence the tumor cell microenvironment (16). Both RAGE and NOXs mediate the chronic inflammatory response, which may potentially increase the risk of carcinogenesis; however, whether there is an association between the factors remains largely unknown. The present study aimed to clarify the possible mechanism of how glucose affects the growth and migration of cancer cells. Therefore, affecting glucose metabolism may be a potential target to suppress cancer cells, which would avoid the side effects that occur with the use of hypoglycemic drugs such as melamine.

\section{Materials and methods}

Cell culture. Human lung adenocarcinoma A549 cells, purchased from Shanghai Institute of Cell Biology of the Chinese Academy of Sciences (Shanghai, China), were cultured in Dulbecco's modified Eagle's medium (DMEM; Servicebio, Inc., Woburn, MA, USA), for $24 \mathrm{~h}$, supplemented with $10 \%$ fetal bovine serum (FBS; Servicebio, Inc.), $25 \mathrm{mmol} / \mathrm{l}$ glucose in the HG group or $5.5 \mathrm{mmol} / 1$ glucose in the normal concentration of glucose (NG) group, and $100 \mathrm{U} / \mathrm{ml}$ penicillin at $37^{\circ} \mathrm{C}$ in a humidified atmosphere containing $5 \% \mathrm{CO}_{2}$. The cells were used between passages 6 and 10 .

NOX inhibitor and RAGE-blocking antibody. The NOX inhibitor diphenyl iodonium chloride (DPI) was purchased from Tocris Bioscience (Bristol, UK; no. 4673-26-1) and was dissolved in dimethyl sulfoxide (DMSO), in order to provide a final concentration of $5 \mu \mathrm{M}$, which was stored at $4^{\circ} \mathrm{C}$. RAGE affinity-purified antibody (5 $\mu \mathrm{g} / \mathrm{ml}$; cat. no. PB0530; Boster Biological Technology, Pleasanton, CA, USA) was dissolved in PBS, and used as a RAGE-blocking antibody.

Cell proliferation and viability. Cell proliferation and viability were assessed using an MTT assay. The cells were seeded at a density of $2 \times 10^{3}$ cells/well (in $5 \% \mathrm{FBS}$ ) or $5 \times 10^{3}$ cells/well (in $0.2 \%$ FBS) onto 96 -well plates in $100 \mu \mathrm{l}$ DMEM overnight, after which the medium was removed. Following treatment with different concentrations of glucose $(0,5,10$ and $25 \mathrm{mmol} / \mathrm{l})$, with or without $5 \mu \mathrm{M}$ DPI or $5 \mu \mathrm{g} / \mathrm{ml}$ RAGE-blocking antibody for $24 \mathrm{~h}, 20 \mu \mathrm{l}$ MTT solution ( $5 \mathrm{mg} / \mathrm{ml}$; Servicebio, Inc.) was added and the cells were cultured for a further $4 \mathrm{~h}$. Subsequently, the medium was removed and $150 \mu \mathrm{l}$ DMSO was added to dissolve the purple formazan for 5-10 min. Absorbance was measured at a wavelength of $555 \mathrm{~nm}$ using an ELISA plate reader.

Cell migration assay. Cell migration was examined by wound-healing assay. Following treatment with $\mathrm{HG}$ or NG, with or without $5 \mu \mathrm{M}$ DPI or $5 \mu \mathrm{g} / \mathrm{ml}$ RAGE-blocking antibody for $24 \mathrm{~h}$, A549 cells were seeded on 6-well plates at a density of $5 \times 10^{3} /$ well and a straight scratch was made using a $200-\mu 1$ sterile pipette tip. Subsequently, the 6-well plates were washed with PBS three times and fresh medium was added. After
$18 \mathrm{~h}$, migration was determined by comparing the wound area between the NG group and the HG group under an inverse fluorescent microscope. Distance change rate was calculated by initial distance-final distance/initial distance.

RNA extraction, cDNA synthesis and $q P C R$. The cells were plated at a density of $1.5 \times 10^{5}$ cells/well in 12-well plates and were cultured for $24 \mathrm{~h}$ after treated with $\mathrm{HG}$ or NG. Total RNA was extracted using RNA rapid extraction solution (Servicebio, Inc.), according to the manufacturer's protocol. The quality and quantity of isolated total RNA were assessed using a NanoDrop ${ }^{\mathrm{TM}} 2000$ Spectrophotometer (NanoDrop; Thermo Fisher Scientific, Inc., Wilmington, DE, USA). RNA was reverse transcribed to cDNA using a RevertAid First Strand cDNA Synthesis kit (Thermo Fisher Scientific, Inc., Waltham, MA, USA) according to the manufacturer's protocol. FastStart Universal SYBR-Green Master (Rox) (Roche Diagnostics, Basel, Switzerland) was used for qPCR analysis, according to the manufacturer's protocol. Fluorescence detection was conducted using an ABI StepOne Plus Real-Time PCR system (Applied Biosystems; Thermo Fisher Scientific, Inc., Waltham, MA, USA). The $2^{-\Delta \Delta \mathrm{Cq}}$ method was used to quantify the expression levels of target genes (17). The following primers were used in the present study (all $5^{\prime} \rightarrow 3^{\prime}$ ): $\beta$-actin forward, CACCCAGCACAATGA AGATCAAGAT and reverse, CCAGTTTTTAAATCCTGA GTCAAGC; RAGE forward, CACTGGTGCTGAAGTGTA AGGG and reverse, CGGACTCGGTAGTTGGACTTG; NOX-4 forward, ATTTAGATACCCACCCTCCCG and reverse, CAC AGTACAGGCACAAAGGTCC; hypoxia-inducible factor- $1 \alpha$ (HIF-1 $\alpha$ ) forward, TGATTGCATCTCCATCTCCTACC and reverse, GACTCAAAGCGACAGATAACACG; and vascular endothelial growth factor (VEGF) forward, GGAGGGCAG AATCATCACGA and reverse, GACTCAAAGCGACAGATA ACACG. Actin RNA levels were used as an endogenous control.

Western blot analysis. The cells were plated at a density of $1.5 \times 105$ cells/well in 6 -well plates and cultured for $24 \mathrm{~h}$. The media were subsequently removed and fresh media containing $\mathrm{NG}$ or HG, with or without DPI $(5 \mu \mathrm{M})$ or RAGE-blocking antibody $(5 \mu \mathrm{g} / \mathrm{ml})$, were added for $24 \mathrm{~h}$. Total protein was extracted with a RIPA Lysis Buffer (Servicebio, Inc.), according to the manufacturer's protocol. Proteins $(20 \mu \mathrm{g})$ were separated by $10 \%$ SDS-PAGE and transferred onto polyvinylidene fluoride membranes. Following blocking by $5 \%$ milk, made with skim milk powder (YIli, China) and Tris Buffered saline Tween buffer ( $0.1 \%$ Tween), at normal temperature for $1 \mathrm{~h}$, monoclonal primary antibodies, namely anti-RAGE antibody (1:500; cat. no. PB0530; Wuhan Boster Biological Technology, Ltd., Wuhan, China), anti-NOX-4 antibody (1:1,000; cat. no. ab109225; Abcam), anti-VEGF antibody (1:500; cat. no. GB11034), anti-HIF-1 $\alpha$ antibody (1:1,000, rabbit; cat. no. GB11031) and anti- $\beta$-actin antibody $(1: 2,000$; cat. no. GB13001-3; all Servicebio, Inc.), were added and incubated overnight at $37^{\circ} \mathrm{C}$. Subsequently, the membranes were washed five times by Tris Buffered saline Tween buffer, and incubated with a goat anti-rabbit immunoglobulin $\mathrm{G}$ horseradish peroxidase-conjugated secondary antibody (1:3,000; cat. no. GB23303; Servicebio, Inc.) for $55 \mathrm{~min}$. The membranes were subsequently washed five times by Tris Buffered saline Tween buffer. Finally, the protein bands were detected using 

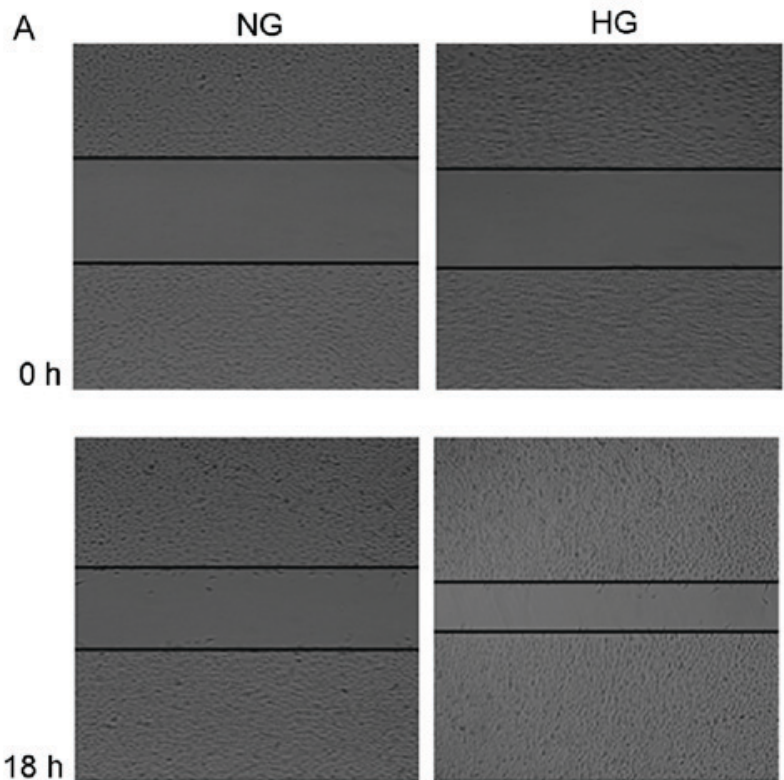

B
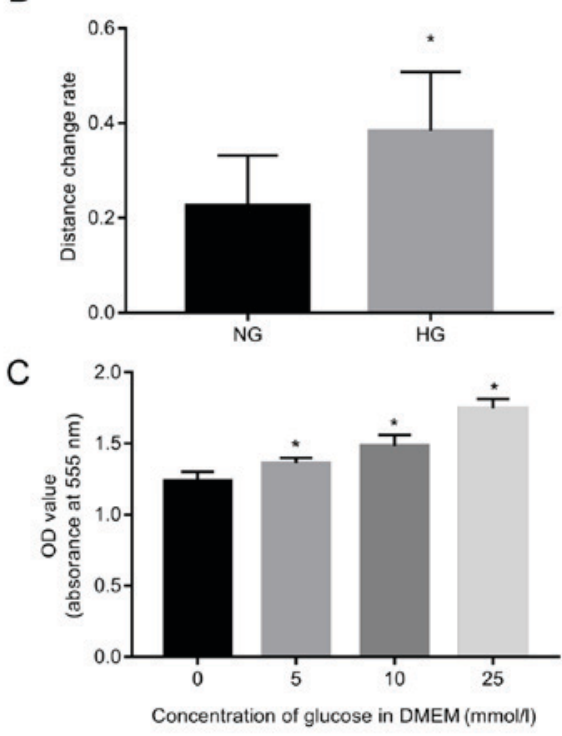

Figure 1. Effects of glucose on migration and proliferation. (A and B) Cell migration could be promoted by HG compared with in cells cultured under NG conditions. (C) MTT results of A549 cells treated with various concentrations of glucose (0, 5, 10 and $25 \mathrm{mmol} / 1)$. Increasing concentrations of glucose promoted the proliferation of A549 cells. "P<0.05 vs. the NG group. DMEM, Dulbecco's modified Eagle's medium; HG, high glucose; NG, normal glucose; OD, optical density.

an EPSON Perfection V300 Photo scanner (Seiko Epson Corporation, Suwa, Japan) after processing with E Enhanced Chemiluminescence visualization reagent (G2014; Servicebio, Inc.).

Statistical analysis. Data are presented as the means \pm standard error of the mean of three repeat. Unpaired t-test was selected to compare two groups, and analyses of multiple groups were performed using one-way analysis of variance, followed by least-significant-difference post hoc test. Analysis was performed using SPSS 22.0 software (IBM Corp., Armonk, NY, USA). $\mathrm{P}<0.05$ was considered to indicate a statistically significant difference.

\section{Results}

$H G$ promotes proliferation and migration of $A 549$ cells, and increases the $m R N A$ and protein expression levels of RAGE and NOX-4. The present study determined how various concentrations of glucose affected the proliferation and migration of A549 cells (Fig. 1). The results demonstrated that A549 cells survived well in response to glucose, and increased proliferation was detected when the concentration of glucose was increased $(\mathrm{P}<0.05$; Fig. $1 \mathrm{~A}$ and $\mathrm{B})$. The migration rate increased significantly in the HG group compared to $\mathrm{NC}(\mathrm{P}<0.05$; Fig. 1C).

Subsequently, $5 \mu \mathrm{g} / \mathrm{ml}$ RAGE-blocking antibody and $5 \mu \mathrm{M}$ DPI were added to the cells, in order to investigate whether RAGE and NOXs serve roles in HG-induced effects (Figs. 2-4). RAGE-blocking antibody inhibited the protein and mRNA expression levels of RAGE and NOX-4 under HG condition, which has the ability to promote the expression of the mRNA and protein of the RAGE and NOX-4 (Fig. 2A and B). Furthermore, treatment with RAGE-blocking antibody accelerated the proliferation $(\mathrm{P}<0.05$; Fig. $3 \mathrm{~A})$ and suppressed HG-induced migration $(\mathrm{P}<0.05 ;$ Fig. $4 \mathrm{~A})$ of A549 cells, thus indicating that
RAGE-blocking antibody may reverse HG-induced effects on cell metastasis. Similar effects were detected in cells treated with the NOX inhibitor DPI; briefly, HG-induced proliferation $(\mathrm{P}<0.05$; Fig. 3B) and migration $(\mathrm{P}<0.05$; Fig. 4B) of A549 cells were inhibited by DPI, which also suppressed the protein expression levels of NOX-4, (Fig. 2E) while having no significant effect on the mRNA expression levels of RAGE (Fig. 2A).

RAGE-blocking antibody and DPI inhibit the expression of HIF-1 $\alpha$ and VEGF, which are involved in mediating tumorigenesis, tumor growth and cancer metastasis. Finally, to determine how the RAGE-NOX-4 pathway affects the biological functions of tumor cells, variations in the expression levels of VEGF and HIF-1 $\alpha$ were measured when RAGE and NOX-4 were suppressed by their respective inhibitors. The mRNA and protein expression levels of VEGF and HIF-1 $\alpha$ were increased under HG conditions compared with NG, whereas treatment with the RAGE-blocking antibody and DPI reduced the expression levels of VEGF and HIF-1 $\alpha$, which indicated that these inflammatory factors may be downstream effectors in the glucose-associated cellular pathways (Fig. 2C-E). However, the mechanism by which the RAGE-blocking antibody and DPI act on VEGF and HIF-1 $\alpha$ in the NG groups, remain unclear.

\section{Discussion}

The constant increase in diabetes mellitus-associated morbidity between 2002 and 2012 indicates that the occurrence of diabetes is associated with a diverse range of complications, which further increases the public health threat posed by this disease (18). Furthermore, recent studies have suggested that a pre-existing hyperglycemic condition, together with the time of diagnosis and insulin deficiency, may exert negative effects on patient prognosis and contribute to the local recurrence of a pulmonary neoplasm by impacting the signaling 
A
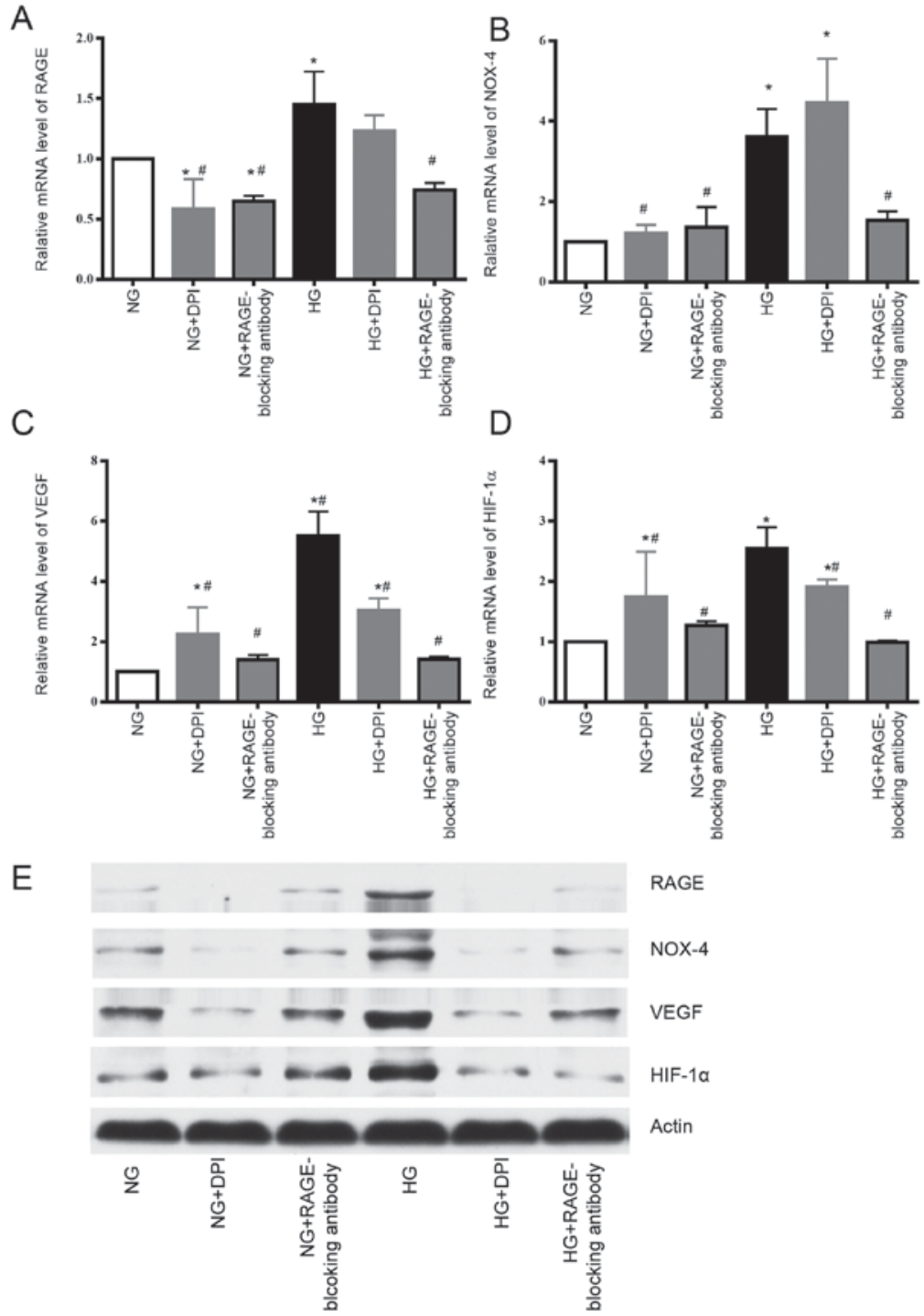

Figure 2. HG increases the mRNA and protein expression levels of RAGE and NOX-4, whereas RAGE-blocking antibody inhibits these effects. DPI could suppress the protein expression of NOX-4 and RAGE induced by HG compared with the HG group. Individual treatment with DPI or the RAGE-blocking antibody decreases the mRNA expression levels of VEGF and HIF-1 $\alpha$ in HG group. The protein expression levels of HIF-1 $\alpha$ and VEGF were also decreased following treatment with the RAGE-blocking antibody or DPI in HG group. (A) RAGE mRNA, (B) NOX-4 mRNA, (C) VEGF mRNA and (D) HIF-1 $\alpha$ mRNA expression was detected by qPCR. (E) RAGE, NOX-4, VEGF and HIF-1 $\alpha$ protein expression was detected by western blotting. ${ }^{*} \mathrm{P}<0.05$ vs. NG group; ${ }^{*} \mathrm{P}<0.05$ vs. HG group. DPI, diphenyl iodonium chloride; HG, high glucose; NG, normal glucose; NOX-4, nicotinamide adenine dinucleotide phosphate oxidase-4; RAGE, receptor for advanced glycation end-products; HIF-1 $\alpha$, hypoxia-inducible factor $1 \alpha$; VEGF, vascular endothelial growth factor; qPCR, quantitative polymerase chain reaction.

pathways of cancer cells $(5,6)$. This viewpoint has been verified by another study, which demonstrated that metformin may improve the chemotherapy outcomes and survival rate of patients presenting with both diabetes and lung carcinoma in a dose-dependent manner (19). The present study confirmed that HG may promote the proliferation and migration of the human NSCLC cell line A549 in a dose-dependent manner, thus indicating that HG may be a risk factor not only for metastasis, but also for the growth of lung adenocarcinoma tumor mass.

RAGE, which is a member of the immunoglobulin superfamily, is a pattern recognition receptor that can bind a diverse range of ligands with similar three-dimensional structures (12). Since the identification of RAGE on endothelial cells, the role of RAGE has gradually been established in certain pathological processes associated with chronic inflammation, including asthma, lung cancer and chronic obstructive pulmonary disease. It has previously been reported that
RAGE exhibits acceleration of the growth and metastasis of pulmonary solid tumor tissue (20). Nevertheless, the signaling pathways of RAGE are yet to be elucidated. The results of the present study demonstrated that RAGE may be a protective factor in the growth of lung adenocarcinoma tumor tissues, but a potential risk factor for metastasis. Furthermore, its expression was increased in response to HG exposure; therefore, it may be hypothesized that RAGE potentially participates in HG-induced oxidative stress via activating NOXs.

NOXs are multi-protein complexes that give rise to the generation of ROS, which can in turn mediate oxidative stress and inflammatory responses (21). The effect of NOXs on ROS generation can also be initiated by HG, thus suggesting that an association may exist between NOXs and HG. In addition, NOXs may facilitate the proliferation and migration of tumor cells by activating certain signaling pathways, including nuclear factor- $\mathrm{\kappa B}$ signaling (22). In the present study, NOX-4, a member of the 

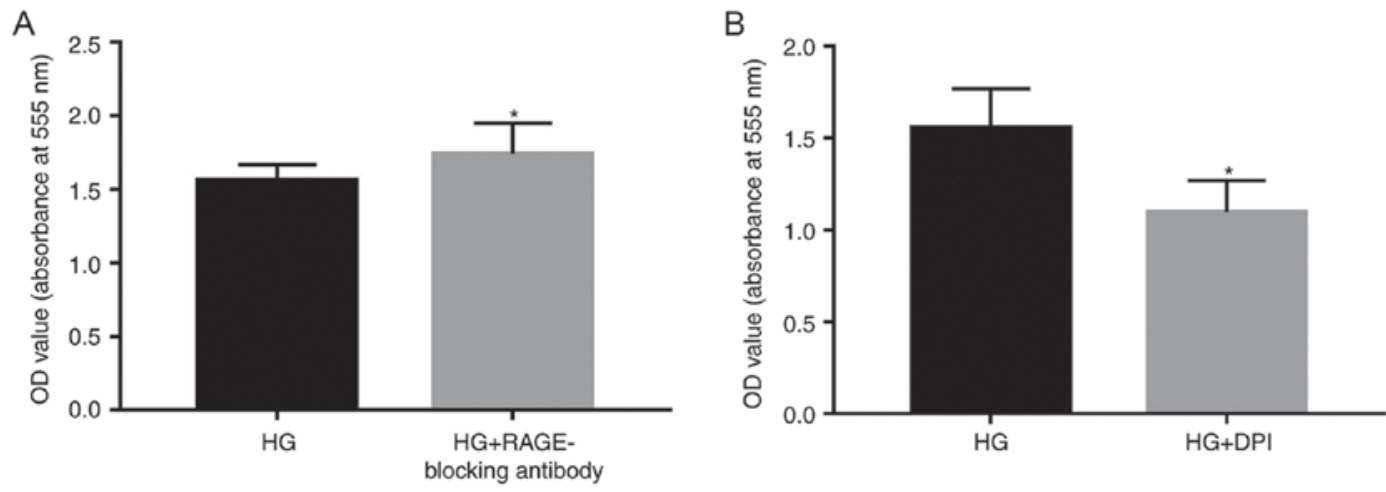

Figure 3. Results of an MTT assay demonstrated that (A) RAGE-blocking antibody promoted HG-induced proliferation of A549 cells, whereas (B) DPI inhibited HG-induced proliferation of A549 cells. "P<0.05 vs. the HG group. DPI, diphenyl iodonium chloride; HG, high glucose; OD, optical density; RAGE, receptor for advanced glycation end-products.
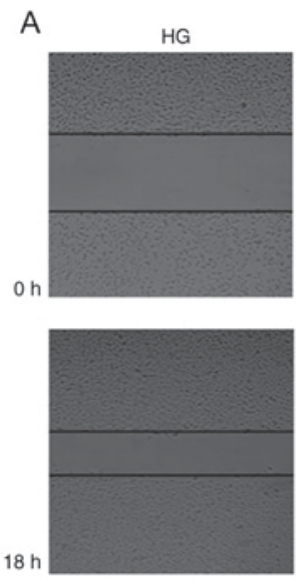

B
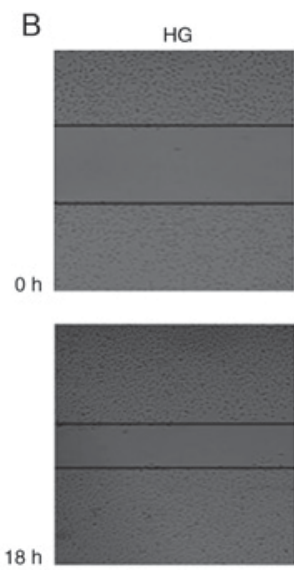

HG+RAGE-

blocking antibody
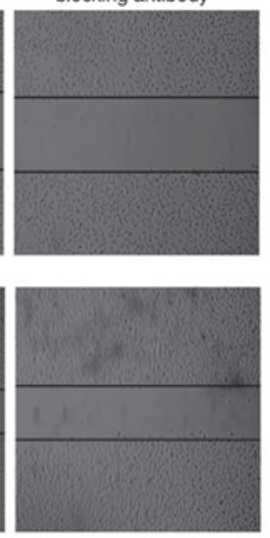

$H G+D P I$
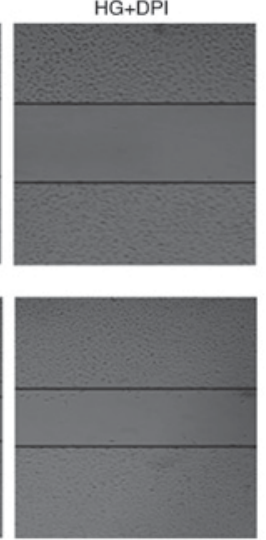
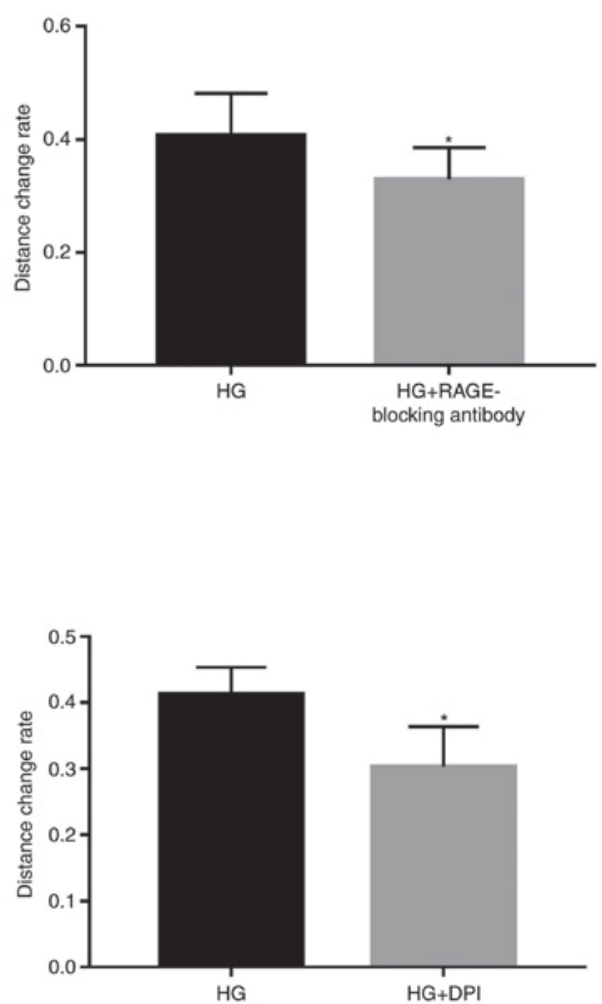

Figure 4. Results of a wound-healing assay indicate that (A) RAGE-blocking antibody may inhibit HG-induced migration of A549 cells. (B) DPI also inhibited HG-induced migration of A549 cells. *P<0.05 vs. the HG group. HG, high glucose; DPI, diphenyl iodonium chloride; RAGE, receptor for advanced glycation end-products.

NOX family, was induced in response to HG; it was suggested that this effect was mediated by RAGE in lung adenocarcinoma cells. Furthermore, the NOX inhibitor DPI served as a protective agent in HG-induced proliferation and migration of A549 cells. These results revealed a potential mechanism underlying HG-induced ROS production via the RAGE-NOX-4 pathway.

Overexpression of HIF-1 $\alpha$ has been observed in various types of solid tumors, and through its corresponding nuclear receptor, HIF-1 $\alpha$ may regulate genes involved in cancer progression, and subsequently promote the proliferation and invasion of lung carcinoma cells (23). VEGF is a protein that mediates angiogenesis and, in turn, sustains the growth of tumor tissue; therefore, the abnormal expression of this factor may have a stimulatory effect on cancer development (24). In addition, VEGF and HIF-1 $\alpha$ are associated with tumor growth and metastasis in various pathways, and are likely associated with the poor prognosis of patients. The present study unveiled a potential approach for controlling the expression of these factors through obstructing the glucose-RAGE-NOX-4 pathway, in order to ultimately inhibit tumor progression. 
In conclusion, abnormal glucose metabolism is a pathological factor facilitating the oncogenic and biological behavior of lung cancer cells, which likely occurs through activation of the RAGE-NOX-4 pathway, and subsequent upregulation of VEGF and HIF-1 $\alpha$. Through binding of these inflammatory factors to their corresponding receptors to exert their specific biological effects, cancer cells may grow faster and their malignancies may be enhanced. Therefore, the significance of controlling blood glucose levels in patients with lung cancer combined with diabetes is evident, and RAGE and/or NOXs may be potential therapeutic targets for the treatment of patients with lung adenocarcinoma and comorbid diabetes. However, the method of administering antagonists rather than performing gene silencing may not be the preferred plan, due to its inconsistent effects during the present study. In addition, why the RAGE-blocking antibody promoted HG-induced proliferation of A549 cells, and whether RAGE-NOXs mediate other effects, as well as inducing the expression of VEGF and HIF-1 $\alpha$, remain unknown; therefore, we aim to address these issues in future research.

\section{Acknowledgements}

Not applicable.

\section{Funding}

The present study was supported by the Wu Jieping Medical Fund of China (grant no. 320.6750.17056).

\section{Availability of data and materials}

The data sets used and/or analyzed during the current study are available from the corresponding author on reasonable request.

\section{Authors' contributions}

YFL and FL designed and conducted the experiment, data analysis, and conclusion completed jointly by YFL, FL and BZ. The manuscript was produced by YFL and then reviewed and revised by FL and BZ.SY and ZQC participated in part of the experimental process.

\section{Ethics approval and consent to participate}

Not applicable.

\section{Consent for publication}

Not applicable.

\section{Competing interests}

The authors declare that they have no competing interests.

\section{References}

1. Zhang Y, Ren JS, Huang HY, Shi JF, Li N, Zhang Y and Dai M: International trends in lung cancer incidence from 1973 to 2007. Cancer Med: Mar 14, 2018 (Epub ahead of print).

2. Cigarette smoking among adults-United States, 2006. MMWR Morb Mortal Wkly Rep 56: 1157-1161, 2007.
3. Morrison HI, Semenciw RM, Mao Y and Wigle DT: Cancer mortality among a group of fluorspar miners exposed to radon progeny. Am J Epidemiol 128: 1266-1275, 1988.

4. Spitz MR, Hong WK, Amos CI, Wu X, Schabath MB, Dong Q, Shete $\mathrm{S}$ and Etzel CJ: A risk model for prediction of lung cancer. J Natl Cancer Inst 99: 715-726, 2007.

5. Luo J, Hendryx M, Qi L, Ho GY and Margolis KL: Pre-existing diabetes and lung cancer prognosis. Br J Cancer 115: 76-79, 2016.

6. Yang X, Liu Y, Mani H, Olson J, Clawson G, Caruso C, Bruggeman R, Varlotto JM, Zander DS and Rassaei N: Biologic evaluation of diabetes and local recurrence in non-small cell lung cancer. Pathol Oncol Res 23: 73-77, 2017.

7. Zhu L, Cao H, Zhang T, Shen H, Dong W, Wang L and Du J: The effect of diabetes mellitus on lung cancer prognosis: A PRISMA-compliant meta-analysis of Cohort Studies. Medicine (Baltimore) 95: e3528, 2016.

8. Iachina M, Jakobsen E, Møller H, Lüchtenborg M, Mellemgaard A, Krasnik M and Green A: The effect of different comorbidities on survival of non-small cells lung cancer patients. Lung 193: 291-297, 2015.

9. Lin JJ, Gallagher EJ, Sigel K, Mhango G, Galsky MD, Smith CB LeRoith D and Wisnivesky JP: Survival of patients with stage IV lung cancer with diabetes treated with metformin. Am J Respir Crit Care Med 191: 448-454, 2015.

10. Kang X, Kong F, Wu X, Ren Y, Wu S, Wu K, Jiang Z and Zhang W: High glucose promotes tumor invasion and increases metastasis-associated protein expression in human lung epithelial cells by upregulating heme oxygenase-1 via reactive oxygen species or the TGF- $\beta 1 /$ PI3K/Akt signaling pathway. Cell Physiol Biochem 35: 1008-1022, 2015.

11. Fritz G: RAGE: A single receptor fits multiple ligands. Trends Biochem Sci 36: 625-632, 2011.

12. Oczypok EA, Perkins TN and Oury TD: All the 'RAGE' in lung disease: The receptor for advanced glycation endproducts (RAGE) is a major mediator of pulmonary inflammatory responses. Paediatr Respir Rev 23: 40-49, 2017.

13. Wang H, Li Y, Yu W, Ma L, Ji X and Xiao W: Expression of the receptor for advanced glycation end-products and frequency of polymorphism in lung cancer. Oncol Lett 10: 51-60, 2015.

14. Hollenbach M: The role of Glyoxalase-I (Glo-I), Advanced Glycation Endproducts (AGEs), and their receptor (RAGE) in chronic liver disease and Hepatocellular Carcinoma (HCC). Int J Mol Sci 18: pii: E2466, 2017.

15. Tang CT, Lin XL, Wu S, Liang Q, Yang L, Gao YJ and Ge ZZ: NOX4-driven ROS formation regulates proliferation and apoptosis of gastric cancer cells through the GLI1 pathway. Cell Signal 46: 52-63, 2018.

16. Kim E, Kim W, Lee S, Chun J, Kang J, Park G, Han I, Yang HJ, Youn $\mathrm{H}$ and Youn B: TRAF4 promotes lung cancer aggressiveness by modulating tumor microenvironment in normal fibroblasts. Sci Rep 7: 8923, 2017.

17. Livak KJ and Schmittgen TD: Analysis of relative gene expression data using real-time quantitative PCR and the 2(-Delta Delta C(T)) method. Methods 25: 402-408, 2001.

18. Mayer-Davis EJ, Dabelea D and Lawrence JM: Incidence trends of type 1 and type 2 diabetes among youths, 2002-2012. N Engl J Med 377: 301, 2017.

19. Mazzone PJ, Rai H, Beukemann M, Xu M, Jain A and Sasidhar M: The effect of metformin and thiazolidinedione use on lung cancer in diabetics. BMC Cancer 12: 410, 2012.

20. Yu YX, Pan WC and Cheng YF: Silencing of advanced glycosylation and glycosylation and product-specific receptor (RAGE) inhibits the metastasis and growth of non-small cell lung cancer. Am J Transl Res 9: 2760-2774, 2017.

21. Souabni H, Ezzine A, Bizouarn T and Baciou L: Functional assembly of soluble and membrane recombinant proteins of mammalian NADPH oxidase complex. Methods Mol Biol 1635: 27-43, 2017.

22. Auer S, Rinnerthaler M, Bischof J, Streubel MK, Breitenbach-Koller H, Geisberger R, Aigner E, Cadamuro J, Richter K, Sopjani M, et al: The human NADPH oxidase, Nox4, regulates cytoskeletal organization in two cancer cell lines, HepG2 and SH-SY5Y. Front Oncol 7: 111, 2017.

23. Yang N, Liang Y, Yang P and Ji F: Propofol suppresses LPS-induced nuclear accumulation of HIF-1 $\alpha$ and tumor aggressiveness in non-small cell lung cancer. Oncol Rep 37: 2611-2619, 2017.

24. Frezzetti D, Gallo M, Maiello MR, D'Alessio A, Esposito C, Chicchinelli N, Normanno N and De Luca A: VEGF as a potential target in lung cancer. Expert Opin Ther Targets 21: 959-966, 2017. 